DOI: $10.1002 /(($ please add manuscript number $))$

Article type: Communication

\title{
Low Thermal Conductivity through Dense Particle Packings with Optimum Disorder
}

Fabian A. Nutz ${ }^{\dagger}$, Alexandra Philipp ${ }^{\dagger}$, Bernd A. F. Kopera, Martin Dulle, Markus Retsch*

Prof. Dr. Markus Retsch, Fabian A. Nutz, Alexandra Philipp, Bernd A. F. Kopera

University of Bayreuth, Department of Chemistry, Universitaetsstr. 30, 95447 Bayreuth, Germany

E-mail: markus.retsch@uni-bayreuth.de

Dr. Martin Dulle

JCNS-1/ICS-1: Neutron Scattering, Forschungszentrum Jülich GmbH, Wilhelm-JohnenStraße, 52428 Jülich

${ }^{\dagger}$ these authors contributed equally

Keywords: colloidal glass; thermal transport; photonic crystal; next neighbor correlation; amorphous limit

Materials with low thermal conductivity are employed in many fields such as clothing, refrigeration, building insulation, and thermoelectrics. ${ }^{[1]}$ Concepts to reduce the thermal conductivity of a given material either target the material composition or its nano-, meso-, or microstructure. Generally, for bulk materials, a high thermal conductivity is found in crystalline solids where phonons are able to transport thermal energy over several hundred nanometers based on a well-defined crystal lattice. ${ }^{[2-5]}$ In contrary, low thermal conductivities are mostly found in disordered, amorphous materials. Based on an increased scattering at the disordered structure, the mean free path of phonons is strongly reduced, resulting in diffusive thermal transport. ${ }^{[6,7]}$ Allen and Feldman ${ }^{[8,9]}$ proposed three different vibrational modes, namely diffusons, propagons, and locons, to describe thermal transport in amorphous matter. Increasing phonon scattering in bulk materials, subsequently leads to a reduction in thermal conductivity. In dense materials this can be achieved by layered structures, ${ }^{[10,11]}$ doping ${ }^{[12,13]}$ or the embedding of nanoparticles, ${ }^{[14,15]}$ where particularly crystalline solids are strongly affected. Zhang and Minnich investigated how nanoparticle clusters with a particular size 


\section{WILEY-VCH}

distribution can lower the thermal conductivity in crystalline SiGe alloys even below its amorphous limit. $^{[14]}$

Besides the chemical composition of a certain material, the influence of the overall structure is of high importance, too. The most obvious and well-known structural influence is given by the introduction of porosity. This severely decreases the density of a bulk material and consequently reduces the effective thermal conductivity. Depending on the cell size of the porous material, highly insulating properties are accessible. ${ }^{[16-19]}$ Moreover, classical porous, granular matter becomes increasingly important for a range of applications, which involve heterostructures and interfaces. For instance, thermal management in high-density storage devices such as batteries or supercapacitors is a critical safety concern. ${ }^{[20-22]}$

Colloidal assembly structures have been proven to be particular suitable to investigate the influence of three-dimensional nanostructuring on the effective thermal transport in granular matter. ${ }^{[23]}$ The thermal conductivity of colloidal crystals is mainly governed by the geometrical constrictions at the interparticle contact points, and the material of the particles itself. ${ }^{[24-26]}$ While most colloidal assembly structures targeted periodically ordered materials, the controlled introduction of disorder also showed to provide fundamental insights, for instance into the physics of phononic bandgaps in the hypersonic regime. ${ }^{[27]}$ In this work, we provide a conceptual understanding of the influence of structural order on the thermal transport properties in particulate mesostructures. Therefore, binary colloidal assemblies were fabricated from two monodisperse, differently sized polystyrene (PS) particles. These buildings blocks are easily accessible by common polymerization techniques ${ }^{[28,29]}$ and their self-assembly into superstructures is well-established. ${ }^{[30]}$ By mixing two differently sized particles, it is possible to control the structural order of the resulting colloidal assembly. We clarify the underlying effect of the thermal conductivity reduction by finite element modeling combined with molecular dynamic simulations. We further demonstrate how to reduce heat transport in disordered binary assemblies in a rational way. 


\section{WILEY-VCH}

Binary colloidal assemblies of PS particles possessing a diameter of $243 \mathrm{~nm}(\mathrm{~S})$ and $306 \mathrm{~nm}$

(L) were fabricated by evaporation-induced self-assembly (size ratio $\sim 0.8$ ). For spectroscopic characterization, binary assemblies were additionally immobilized on a glass slide by dip-coating of a $3 \mathrm{wt} \%$ of aqueous particles dispersions. The mixing ratio ranged from 0 vol\%-L to 100 vol\%-L. Optical microscopy and scanning electron images of the edges of split monoliths are shown in Figure 1a and $\mathrm{b}$ and provide a qualitative expression of how the mixing ratio translates into order and disorder.

From optical microscopy (Figure 1a), strong opalescent colors are visible for samples consisting of only one particle type ( 0 vol\%-L and 100 vol\%-L) as well as for binary assemblies only containing a small amount of differently sized specimen (9 vol\%-L, 95 vol\%-L, and 97 vol\%-L). This is due to Bragg reflection. The difference in color between 0 vol\%-L and 100 vol\%-L monoliths arises from the size dependence of the Bragg reflectivity. Different colors within one monolith can be attributed to the angle dependence of the Bragg law, based on different crystal planes exposed to the surface.

For other mixing ratios, only weak overall Bragg reflection (66 vol\%-L and 86 vol\%-L) or almost only diffuse scattering (18 vol\%-L and $40 \mathrm{vol} \%-\mathrm{L})$ is visible. At these mixing ratios, the polydispersity of the binary particle dispersion prevents large area particle crystallization. Noteworthy, some embedded crystalline regions are observable for several intermediate mixing ratios (40 vol\%-L, 66 vol\%-L, and 86 vol\%-L). This can be explained by the strong tendency of demixing in binary colloidal dispersions. ${ }^{[31-33]}$ The slow self-assembly process (several days) of the dispersion enables the formation of phase separated, multicrystalline, and amorphous areas.

Scanning electron microscopy (SEM) images validate the optical impression (Figure 1b). The pure colloidal crystals feature a high degree of crystalline particle ordering. In contrast, for intermediate mixing ratios, only a random packing of the particles is observed. This is additionally highlighted by fast Fourier transformation (FFT) images of the SEM images 


\section{WILEY-VCH}

illustrated in Figure 1d. Whereas distinct reflexes are visible for 100 vol\%-L due to the hexagonal symmetry of the particle lattice, only smeared Debye-Scherrer rings are obtained for 18 vol\%-L monoliths. The smearing is based on the disordered mesostructure whereas the observed Debye-Scherrer rings arise from the different particle diameters present within the monoliths.

We further quantify the intrinsic order of the binary colloidal films by UV-vis transmission spectra of dip-coated samples, displayed in Figure 1c. A strong Bragg peak is visible for $0 \mathrm{vol} \%-\mathrm{L}$ colloidal crystals at a wavelength of $559 \mathrm{~nm}$. At small mixing ratios of 9 vol\%-L, the Bragg peak broadens and the maxima is less allocable due to the disturbance of the crystal lattice. A further increase of the large particle volume fraction to $18 \mathrm{vol} \%$-L leads to a vanishing of the Bragg peak. No distinct Bragg peaks are observable for intermediate mixing ratios from 44 vol\%-L to 86 vol\%-L, indicating a randomly packed structure. The Bragg peak of the larger particle species reoccurs at $95 \mathrm{vol} \%$-L and reaches a maximum intensity for $100 \mathrm{vol} \%-\mathrm{L}$ at a wavelength $667 \mathrm{~nm}$.

Optical microscopy, SEM, and UV-vis characterization prove the highly crystalline nature of the homo-particle ensembles, which define the edges of the mixture phase diagram. The long-range order is quickly lost, when mixing two particles with a size ratio of 0.8 . Depending on the assembly process a complete suppression of crystallinity can be found (dip-coating) or small, multicrystalline regions caused by demixing prevail (dispersion drying). Laser flash analysis (LFA) was performed on colloidal monoliths obtained from evaporation-induced self-assembly. By determining the sample density and specific heat capacity, it is possible to calculate the specimen's effective thermal conductivity (details see supporting information). The thermal conductivity, normalized to the maximum initial value of the pure colloidal assemblies, as well as the normalized thermal conductivity of intermediate mixing ratios are show in Figure 2a. The color code helps to separate ordered 


\section{WILEY-VCH}

samples (blueish) from randomly packed assemblies (greenish) and correspond to the mixing ratios given in Figure 1.

Homo-particle colloidal assemblies (mixing ratio 0 vol\%-L and 100 vol\%-L) show a thermal conductivity of $73 \mathrm{mWm}^{-1} \mathrm{~K}^{-1}$ and $77 \mathrm{mWm}^{-1} \mathrm{~K}^{-1}$ in absolute numbers, respectively. At small mixing ratios of differently sized particles, the thermal conductivity slightly drops to $~ 93 \%$ of the initial value. For intermediate mixing ratios, the thermal conductivity drops significantly to $\sim 80 \%$. This reduction can only partially be explained by the reduced density of the intermediate assemblies as displayed in Figure $2 b$. The reduction of the density for intermediate mixing ratios originates from the prevented close packing of the particles during assembly. This leads to a space filling which deviates from the maximum of $74 \%$. Still, the density only decreases to $\sim 90 \%$ of its initial value. Therefore, an additional effect is present, which is responsible for the overall reduction of the thermal conductivity to $80 \%$. To elucidate this additional contribution, we performed finite element modeling (FEM) on binary particle mixtures possessing a size ratio of 0.8 . The required virtual assemblies were obtained by molecular dynamic (MD) simulations of the assembly process. Exemplary particle assemblies obtained from MD simulations are shown in Figure S2a. We found highly crystalline particle assemblies at the edges of the mixing diagram. In contrast to our experimental findings, the crystallization process is already fully prevented at very small mixing ratios (Figure S2a, left). This is caused by the lack of demixing or phase separation of the particles due to the short assembly time-scale used for the MD simulation. FFT images of $0 \mathrm{vol} \%$-L and $9 \mathrm{vol} \%-\mathrm{L}$ assemblies from MD simulation are compared to the experiment in Figure S2a, right. Thus, the fully amorphous structure obtained from the MD simulation can be regarded as an ideally disordered case. Experimentally, some degree of crystallization will be difficult to exclude owing to the long timescale allowed for the self-assembly process. FEM has been conducted using the software COMSOL Multiphysics®. Details on the modeling are described in the Supporting Information. The calculated thermal conductivity 


\section{WILEY-VCH}

against the volume fraction of large particles is displayed in Figure 2c. Analogous to Figure 2a, the color code indicates ordered (bluish) and disordered assemblies (green). The mixing diagram shows a similar trend for the thermal conductivity as observed for the experiment, with high thermal conductivities for ordered, and a reduced thermal conductivity for disorder assemblies. A minimum thermal conductivity of only $55 \%$ of the initial value is observed at a volume fraction of $\sim 16 \mathrm{vol} \%$-L. This is an almost two-fold enhancement of the insulation capability caused by the disordered structure, and it is significantly lower compared to the experimental results. We ascribe this deviation to the structural difference between the MD simulation and the experiment. As outlined before, by MD simulation one can observe a more disordered distribution of both particle types within the monolith, leading to a stronger reduction of the thermal conductivity.

To explain the origin of the thermal conductivity reduction beyond the decrease in density, we utilized FEM to analyze the length of the thermal transport pathway through such a particulate assembly structure. This can be done by evaluating the length of heat flux streamlines, which reach from the hot to the cold side of the simulation box. Figure $2 \mathrm{~d}$ shows two individual cases through an ordered (100 vol\%-L) and disordered ( 16 vol\%-L) particle ensemble. Whereas in the ordered case, a rather straight and unperturbed streamline is obtained, the introduction of disorder strongly bents and perturbes the thermal transport path. The statistic evaluation over the entire simulation box is given in Figure 2e, where we normalized the streamline length on the length of the simulation box. One finds a direct correlation between the streamline length and the thermal conductivity reduction. This finding can be intuitively explained by a sort of effective thermal length, which differs from the plain geometric size of the box. One may draw an analogy to the concept of the diffusive optical path in a particulate powder, which is governed by the scattering cross section of the constituting sphere. ${ }^{[34]}$ Most interestingly, the FEM simulation finds a strongly asymmetric shape of the thermal conductivity reduction with two minima at moderately high and low mixing ratios, 


\section{WILEY-VCH}

respectively. These are not found in the experiment, presumably due to the presence of mesocrystalline domains, embedded in the amorphous structure. We now want to understand the origin of these minima and how the thermal conductivity could be reduced even further. This question could be rephrased to how the thermal streamline length can be further increased in such colloidal superstructures. We, therefore, return to MD simulation and evaluate the local structure around one particle more explicitly. We did this in a twofold way: 1) Using the radial distribution function we evaluate the number of particles surrounding a center particle, which are within the local space of about $10 \%$ of the respective particle diameter. 2) Using the individual coordinates of each particle pair, we extracted the number of touching spheres around a central particle. We term the first one "next neighbors", and the second one "contact neighbors". Quite expectedly, when introducing disorder the average number of next and contact neighbors is reduced. For crystalline assemblies, one can find an average number of next neighbors per particles of $\sim 12$, based on the face centered-cubic symmetry. For all disordered intermediate mixing ratios, the number of next neighbors is reduced to $\sim 10$ (Figure S2b). The next contact neighbor analysis yields $\sim 8$ owing to the more rigorous exclusion criterion. One can imagine that this reduction leads to less pathways for heat to travel through the particle structures and therefore reduces the thermal conductivity of the assemblies. However, we find that the mean number of next neighbor particles merely reaches a broad plateau, and does not explain the asymmetric shape shown in Figure 2c. We, therefore, evaluated the next neighbor particle histograms explicitly. This is shown in Figure 3, where we present the next neighbor histograms at selected mixing ratios (bottom row). We chose a special illustration, to highlight the structural heterogeneity, when heat travels through such a system. We, therefore, present a projection of all particles with the same number of next neighbors that can be found within the simulated box. The color code indicates particles possessing the same number of next neighbors from high (blue) to low (red). It therefore provides a direct impression of how many particles are clustered around one central sphere. 


\section{WILEY-VCH}

For mixing ratios of $0 \mathrm{vol} \%-\mathrm{L}$ and 100 vol $\%$-L almost every particle possesses twelve next neighbors. This indicates a nearly fully crystalline face-centered cubic assembly, with few line and point defects being visible. The particle neighbor distribution function quickly broadens at intermediate mixing ratios. Most importantly, this distribution function shows a strongly asymmetric behavior. For mixing ratios of 9 vol\%-L to 66 vol\%-L, even more than twelve next neighbors per particles are present within the assembly, whereas for high mixing ratios, the number of next neighbors is limited to twelve. For a low number of large particles (small mixing ratios), it is possible that one large particle is decorated by a high number of smaller particles. In contrary, for high mixing ratios of large particles, it is geometrically not possible to decorate a small particle with a high number of large particles, since there is simply not sufficient space. This asymmetry is less pronounced when considering the contact neighbors (Figure S5). The average contact number stays constant with a tendency towards fewer contact points at the minimum volume fraction $\sim 20$ vol\%-L. This discrepancy between a constant or reduced number of contact neighbor particles compared to the evolution of many next neighbor particles hints towards the importance of a jammed particle corona in close vicinity to the central bead.

We also conducted a thorough analysis of the interparticle constriction effect in the Supporting Information (Figure S6-8). We, therefore, analyzed the average interparticle contact area and relate this to the contact neighbor distribution. Using an ideal face-centered cubic reference system we show that a reduction of the interparticle contact area leads to an increase in thermal path length caused by a stronger bending of the heat flux stream lines (Figure S6b). However, the changes of the interparticle contact area play a secondary role compared to the introduction of disorder and consequently to the thermal path length.

The consequence of the asymmetric next neighbor distribution for the effective thermal length is rather surprising. A reduction of the number of next neighbor particles indeed leads to a reduction in thermal conductivity. However, even more efficient is the introduction of a 


\section{WILEY-VCH}

certain amount of many jammed and not necessarily directly contacting particles, since this leads to an even stronger disturbance of the streamline length due to a geometric extension of the predetermined thermal transport path. This is accompanied by a minimum of the total number of interparticle contact points within the simulation volume (Figure S5).

To clarify how this transport pathway is governed by the size mismatch of the two particles, we varied the size ratio of the particles between 0.9 and 0.54 at the mixing ratio around 20 vol\%-L, which showed the lowest thermal conductivity. Noteworthy for this data set, the experimental samples were prepared by filtration to speed up the assembly time and therefore obtain particle structures, which are more comparable to the MD assemblies. Both, experiment and FEM simulation, show a systematic decrease of the thermal conductivity with a decreasing size ratio from 0.9 to 0.54 (Figure $4 \mathrm{~d}$ ). The increase of the thermal conductivity for the smallest size ratio (0.54) for the experiment can be explained by an increasing tendency to demix as outlined above. One also needs to take into account that fully crystalline binary assemblies can be obtained for size ratios of $<0.41$ owing to the octahedral voids in face-centered cubic structures, which we approach with this small size ratio. ${ }^{[35]}$ Figure 4a compares the heat flux density of the particle assemblies from size ratios of 0.54 , 0.8 , and, 0.9 to the heat flux density of a fully crystalline assembly (size ratio: 1.0 ). Crystalline assemblies feature uniform and straight streamlines (Figure 4a, 1.0), whereas a decreasing size ratio evokes a high degree of distortion. This is especially obvious for the heat flux density of the size ratio 0.54 , where one large particle heavily impedes the heat flux through the entire particle box. The qualitative impression from the heat flux density images is verified by comparing the relative streamline lengths of the different size ratios (Figure $4 b$ ). The smaller the size ratio, the higher the number of long streamlines, which can reach up to $140 \%$ relative to the length of the simulation box. This indicates that the less the particle sizes match, the stronger the heat flux is bent along the particle network, leading to an elongation of the thermal transport path. 


\section{WILEY-VCH}

The higher fraction of long streamlines coincides with a broadening of the next neighbor distribution function with decreasing size ratio (Figure 4c). The broadening increases the number of $>10$ next neighbors, indicating an increasing decoration of a large particle with small particles. However, due to the small number of large particles at this volume fraction and size ratio combination (about $4 \%$ number ratio of large particles) this effect is not visible in the average number of neighbors for the whole assembly. Only when looking at the detailed next neighbor histograms the results from the heat flux simulations can be understood (Figure 4c). For a size ratio of 0.54 a second fraction with even 25-30 next neighbors is found. Looking at the contact neighbor distribution for this sample, the number of touching spheres remains rather constant at about 8 (Figure S5). This highlights the evolution of a denselyjammed corona, where a small amount of large particle clusters are decorated with smaller spheres. This constitutes an efficient way to increase the thermal path length. Overall, the higher mismatch between the two particle sizes led to a thermal conductivity reduction by $40 \%$ (size ratio 0.6 ) in the experiment and $53 \%$ (size ratio 0.54 ) in the FEM simulation. We finally also checked, whether 19 vol\%-L represents the absolute minimum with respect to the mixing ratio. We, indeed, find the highest fraction of $>25$ next neighbor particles for this mixing ratio (Figure $\mathrm{S} 4)$. Higher or lower mixing ratios ( \pm 5 vol\%-L) lead rapidly to a reduced number of next neighbors. Therefore, a mixing ratio of $19 \mathrm{vol} \%$-L of large particles is sufficient to strongly reduce the effective thermal transport across a binary particle mixture. We finally compare this disorder derived thermal insulation effect to the thermal conductivity of known polymer materials. The unique properties of colloidal crystals and especially colloidal glasses can be inferred from the Ashby plot in Figure 5 in a straight-forward manner. ${ }^{[36]}$ It shows a direct relation between the thermal conductivity and the polymer density. Using polymer foams, highly insulating materials are accessible, however, at the expense of the materials density. Transitioning from colloidal crystals to colloidal glasses, 


\section{WILEY-VCH}

retains the materials density to a large extent, while the thermal conductivity decreases. This opens the avenue to design highly insulating granular materials at a retained high density. In summary, we investigated the influence of order and disorder in particle mixtures on their thermal transport properties. We therefore choose a controlled mixture of binary latex particles as a model system. In a combined approach, which comprised experimental characterization, MD simulation as well as FEM, we were able to relate the observed reduction in thermal conductivity to the local environment of the colloidal particles. Quite surprisingly, the increase of next neighbor contact points at low mixing ratios and at a high size mismatch between the binary spheres, represents the most efficient way to attain a minimum thermal conductivity. This is achieved at a comparatively high density. The decisive concept is the increase in the effective thermal length, across which the thermal energy needs to be transported. Overall, disorder in a binary colloidal glass can reduce the thermal conductivity by as much as $\sim 50 \%$ compared to its single phase, crystalline counterpart. Our findings are of high importance for heterostructured and particulate materials, for applications were a high insulation, but also a good heat dissipation are paramount. Owing to the diffusive thermal transport in our system, our findings are of conceptual nature and applicable to many other nano-, micro- and macrostructured materials, beyond simple latex beads. Most importantly, we want to stress the relevance of the concept of an effective thermal length, which cannot only be increased by constriction but may be even more strongly affected by additional pathways and detours.

\section{Experimental Section}

Details on the particle synthesis, binary colloidal self-assembly methods, and characterization methods, as wells as a detailed description on thermal transport characterization by laser flash analysis, molecular dynamics simulations, finite element modeling, complementary contact 


\section{WILEY-VCH}

particle analysis and a discussion on the influence of the particle contact area can be found in the Supporting Information.

\section{Supporting Information}

Supporting Information is available from the Wiley Online Library or from the author.

\section{Acknowledgements}

We thank Ute Kuhn for help with DSC measurements. Tobias Lauster, Julian Sindram, and Dominik Skrybeck are acknowledged for performing firsts UV-Vis and LFA experiments.

B.A.F.K. acknowledges support from the Elite Network of Bavaria (ENB). Additional support was provided by the SFB840 and the Bavarian Polymer Institute, BPI. This work was funded by the Volkswagen foundation through a Lichtenberg professorship.

Received: ((will be filled in by the editorial staff))

Revised: ((will be filled in by the editorial staff)) Published online: ((will be filled in by the editorial staff))

\section{References}

[1] B. Poudel, Q. Hao, Y. Ma, Y. Lan, A. Minnich, B. Yu, X. Yan, D. Wang, A. Muto, D. Vashaee, X. Chen, J. Liu, M. S. Dresselhaus, G. Chen, Z. Ren, Science 2008, 320, 634.

[2] D. G. Cahill, P. V. Braun, G. Chen, D. R. Clarke, S. H. Fan, K. E. Goodson, P. Keblinski, W. P. King, G. D. Mahan, A. Majumdar, H. J. Maris, S. R. Phillpot, E. Pop, L. Shi, Appl. Phys. Rev. 2014, 1 .

[3] D. G. Cahill, W. K. Ford, K. E. Goodson, G. D. Mahan, A. Majumdar, H. J. Maris, R. Merlin, Phillpot, S. R., J. Appl. Phys. 2003, 93, 793.

[4] D. G. Cahill, R. O. Pohl, Annu. Rev. Phys. Chem. 1988, 39, 93.

[5] M. Maldovan, Nature 2013, 503, 209.

[6] A. Einstein, Ann. Phys. (Berlin) 1911, 340, 679.

[7] D. G. Cahill, S. K. Watson, R. O. Pohl, Phys. Rev. B 1992, 46, 6131.

[8] P. B. Allen, J. L. Feldman, Phys. Rev. B 1993, 48, 12581. 


\section{WILEY-VCH}

[9] P. B. Allen, J. L. Feldman, J. Fabian, F. Wooten, Philos. Mag. B 1999, 79, 1715.

[10] C. Chiritescu, D. G. Cahill, N. Nguyen, D. Johnson, A. Bodapati, P. Keblinski, P. Zschack, Science 2007, 315, 351.

[11] M. D. Losego, I. P. Blitz, R. A. Vaia, D. G. Cahill, P. V. Braun, Nano Lett. 2013, 13, 2215.

[12] J. Moon, A. J. Minnich, RSC Advances 2016, 6, 105154.

[13] H. Mizuno, S. Mossa, J. L. Barrat, Sci. Rep. 2015, 5, 14116.

[14] H. Zhang, A. J. Minnich, Sci. Rep. 2015, 5, 8995.

[15] W. Kim, J. Zide, A. Gossard, D. Klenov, S. Stemmer, A. Shakouri, A. Majumdar, Phys. Rev. Lett. 2006, 96, 045901.

[16] B. Notario, J. Pinto, E. Solorzano, J. A. de Saja, M. Dumon, M. A. Rodríguez-Pérez, Polymer 2015, 56, 57.

[17] M. Koebel, A. Rigacci, P. Achard, J. Sol-Gel Sci. Technol. 2012, 63, 315.

[18] X. Lu, M. C. Arduini-Schuster, J. Kuhn, O. Nilsson, J. Fricke, R. W. Pekala, Science 1992, 255, 971.

[19] S. N. Schiffres, K. H. Kim, L. Hu, A. J. H. McGaughey, M. F. Islam, J. A. Malen, Adv. Funct. Mater. 2012, 22, 5251.

[20] Z. Chen, P. C. Hsu, J. Lopez, Y. Z. Li, J. W. F. To, N. Liu, C. Wang, S. C. Andrews, J. Liu, Y. Cui, Z. N. Bao, Nat. Energy 2016, 1, 15009.

[21] C. Sangrós, C. Schilde, A. Kwade, Energy Technol. 2016, 4, 1611.

[22] C. Masarapu, H. F. Zeng, K. H. Hung, B. Wei, ACS Nano 2009, 3, 2199.

[23] P. Ruckdeschel, A. Philipp, M. Retsch, Adv. Funct. Mater. 2017.

[24] F. A. Nutz, M. Retsch, Phys. Chem. Chem. Phys. 2017, 19, 16124.

[25] F. A. Nutz, P. Ruckdeschel, M. Retsch, J. Colloid. Interf. Sci. 2015, 457, 96.

[26] P. Ruckdeschel, T. W. Kemnitzer, F. A. Nutz, J. Senker, M. Retsch, Nanoscale 2015, 7, 10059. 


\section{WILEY-VCH}

[27] T. Still, W. Cheng, M. Retsch, R. Sainidou, J. Wang, U. Jonas, N. Stefanou, G. Fytas, Phys. Rev. Lett. 2008, 100, 194301.

[28] J. W. Goodwin, J. Hearn, C. C. Ho, R. H. Ottewill, Colloid. Polym. Sci. 1974, 252, 464.

[29] R. H. Ottewill, J. N. Shaw, Kolloid Z. Z. Polym. 1967, 215, 161.

[30] N. Vogel, M. Retsch, C. A. Fustin, A. Del Campo, U. Jonas, Chem. Rev. 2015, 115, 6265.

[31] P. N. Pusey, W. van Megen, Nature 1986, 320, 340.

[32] M. Dijkstra, R. van Roij, R. Evans, Phys. Rev. Lett. 1998, 81, 2268.

[33] A. V. A. Kumar, The Journal of Chemical Physics 2013, 138, 154903.

[34] J. Wang, S. Ahl, Q. Li, M. Kreiter, T. Neumann, K. Burkert, W. Knoll, U. Jonas, J. Mater. Chem. 2008, 18, 981.

[35] Granta design, CES edupack, Cambridge, UK 2015. 
WILEY-VCH

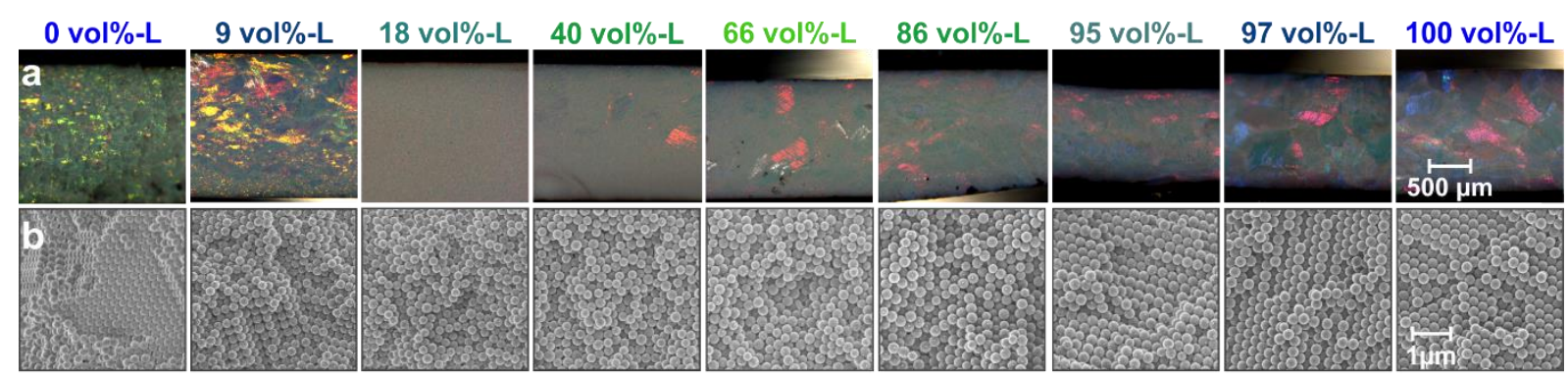

C

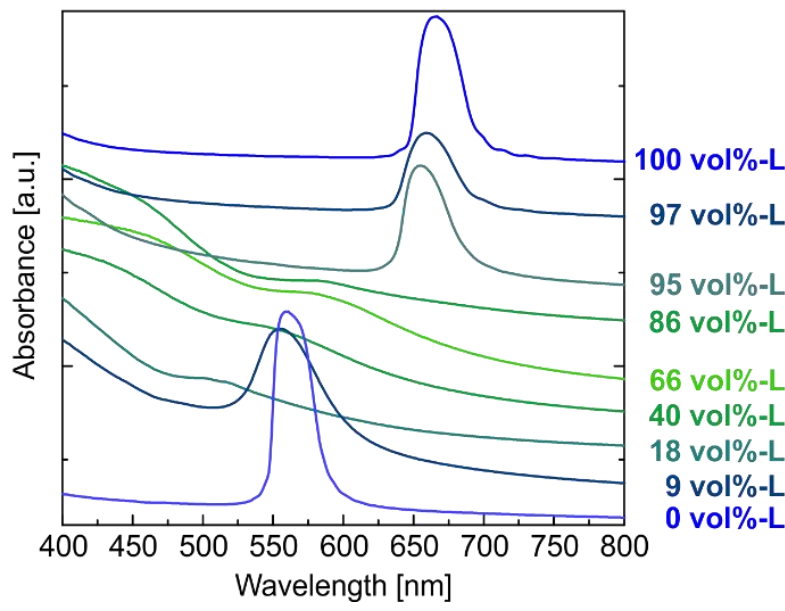

d

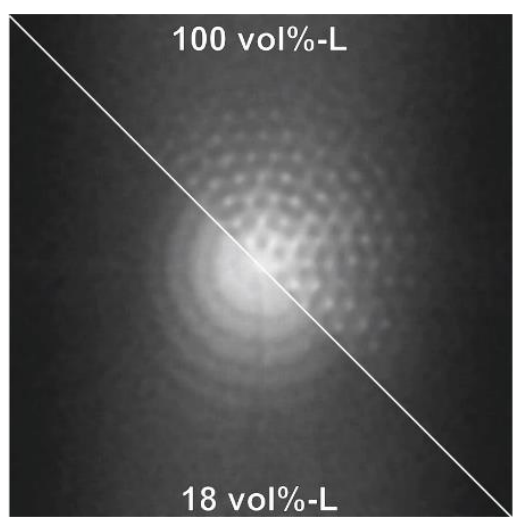

Figure 1. Structural characterization of binary colloidal assemblies. (a) Optical and (b) scanning electron microscopy side view images of the edges of split colloidal monoliths. (c) UV-vis transmission spectra measured on dip-coated assemblies. (d) Fast Fourier transformation (FFT) images from scanning electron micrographs with a mixing ratio of 18 vol\%-L compared to a FFT image of 100 vol\%-L colloidal crystal. 


\section{WILEY-VCH}
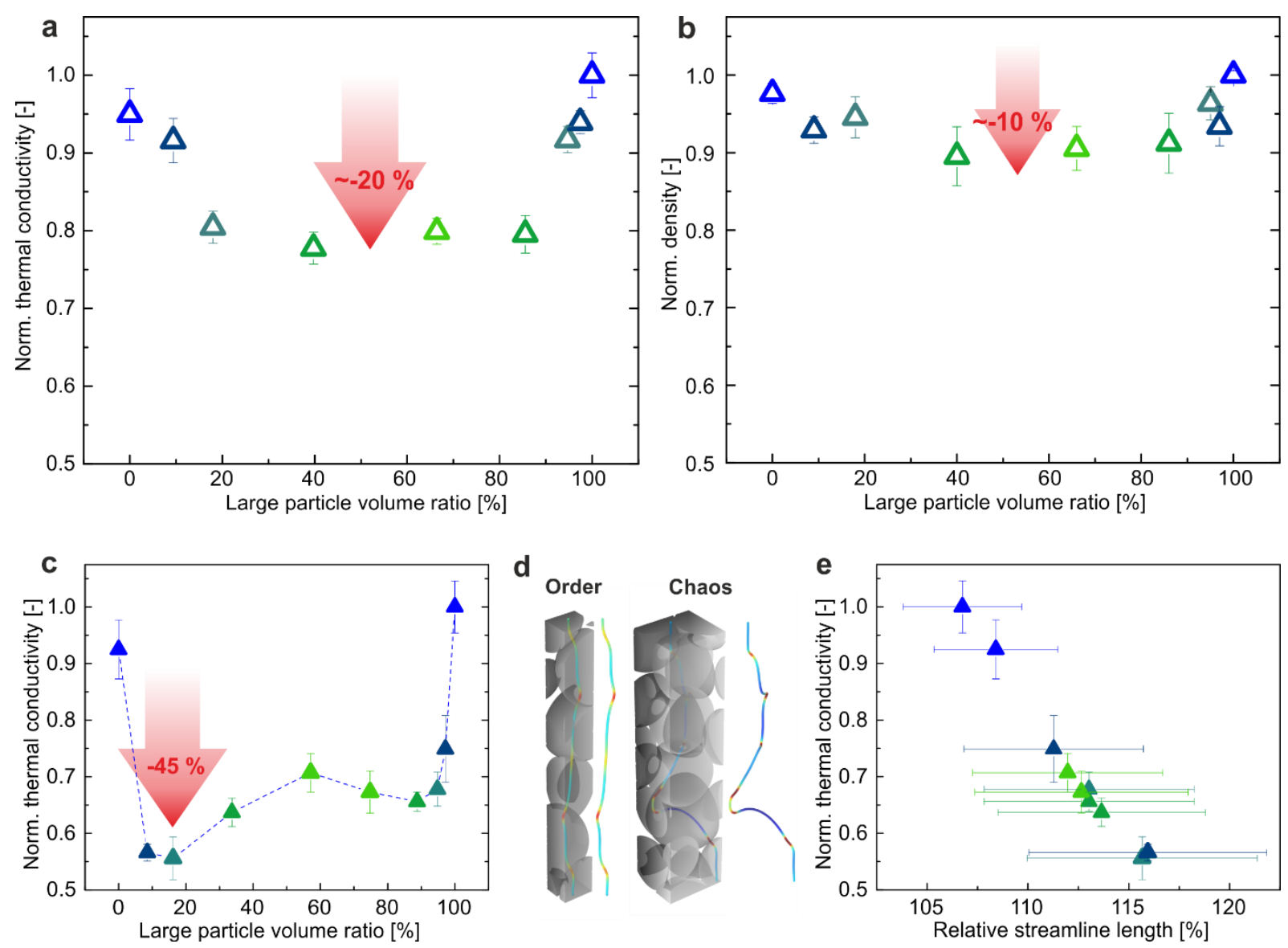

Figure 2. Thermal conductivity of the investigated assemblies with a size ratio of 0.8 . Color code indicates ordered (blueish) and disordered assemblies. (a) Normalized thermal conductivity of binary PS colloidal assemblies possessing different mixing ratios. The red arrow indicates the reduction of the thermal conductivity due to the introduced disorder. (b) Normalized density of the colloidal assemblies. (c) Thermal conductivity vs. volume fraction of large particles obtained by FEM. Error bars arise from three individual simulations boxes at three different directions in space. (d) Exemplary streamlines present within a crystalline and a disordered assembly with a size ratio of 0.8 and 16 vol\%-L. (e) Thermal conductivity vs. relative streamline length of various size ratios. Error bars arise from three individual simulations boxes at three different directions in space. 


\section{WILEY-VCH}

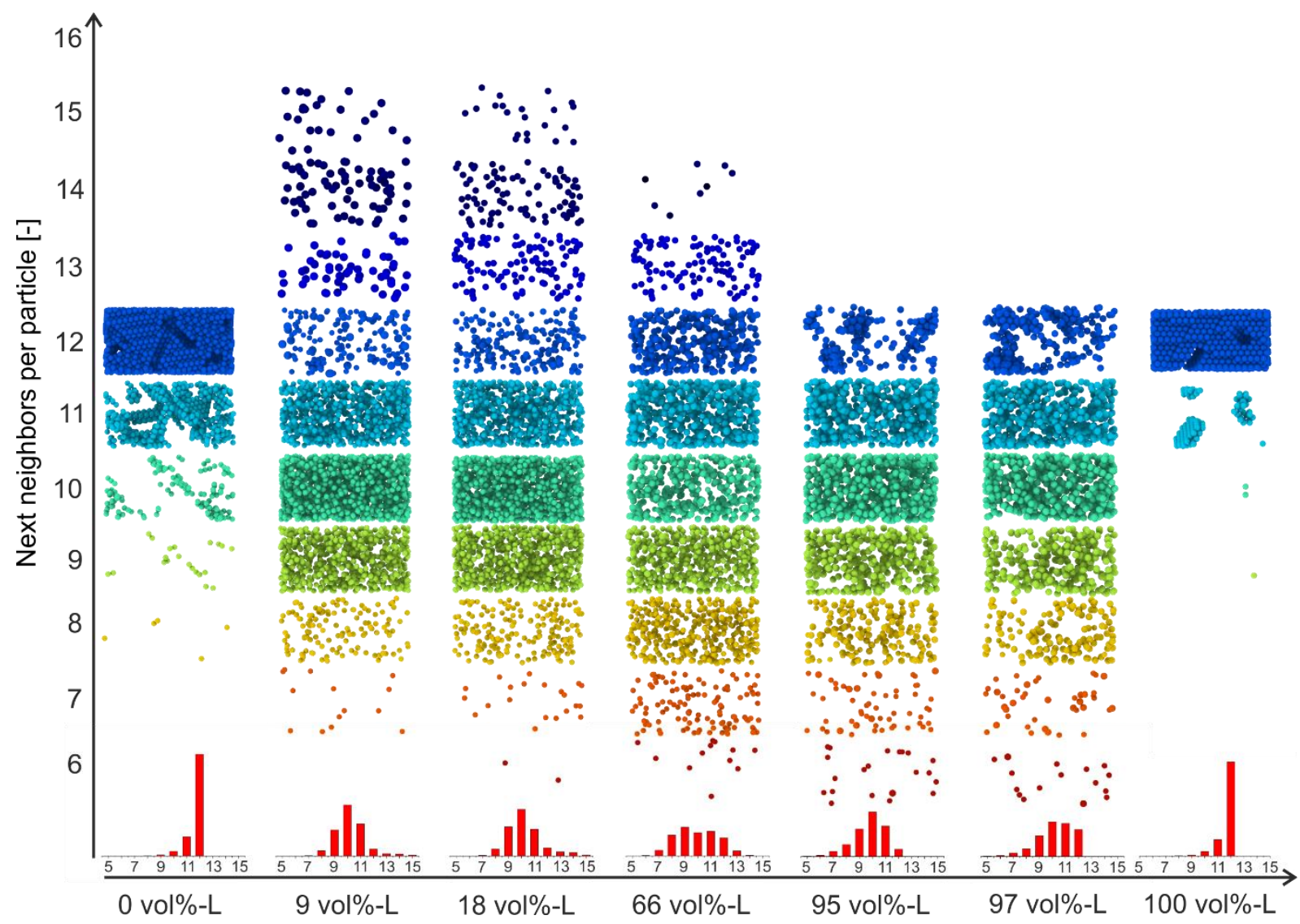

Figure 3. Molecular dynamics simulations of the assembly process of a binary particle mixture. Number of next neighbors per particle against volume fraction of large particles. Size ratio is 0.8 . Projection of the assemblies visualize the particles possessing the indicated number of neighbors. Histograms show the relative frequency of particle neighbors per particle present within the different assemblies. 


\section{WILEY-VCH}

a $D_{\mathrm{s}} / \mathrm{D}_{\mathrm{L}}=0.54$

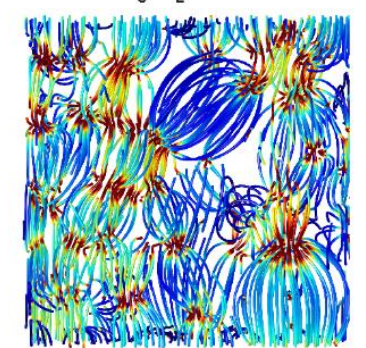

b

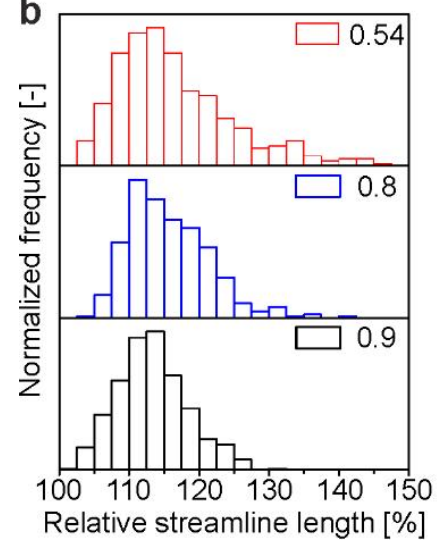

$D_{s} / D_{L}=0.8$

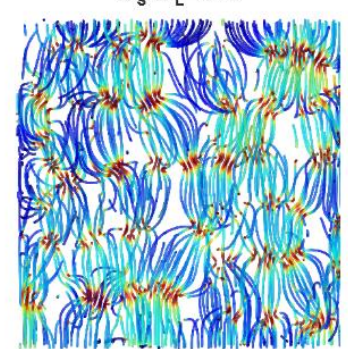

C

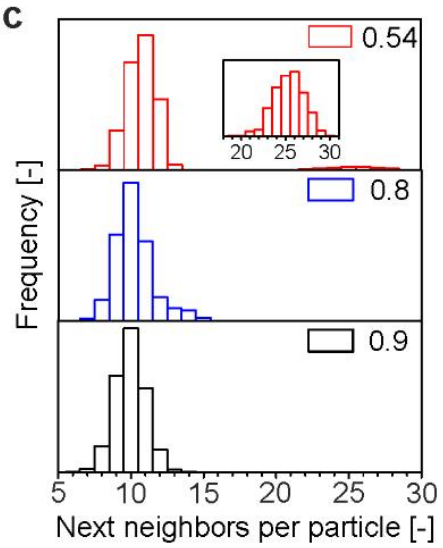

$\mathrm{D}_{\mathrm{s}} / \mathrm{D}_{\mathrm{L}}=0.9$

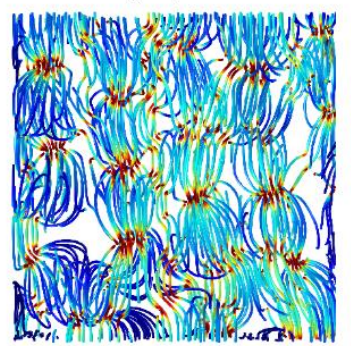

$\mathrm{D}_{\mathrm{s}} / \mathrm{D}_{\mathrm{L}}=1.0 \quad\left[\mathrm{Wm}^{-2} \times 10^{3}\right]$
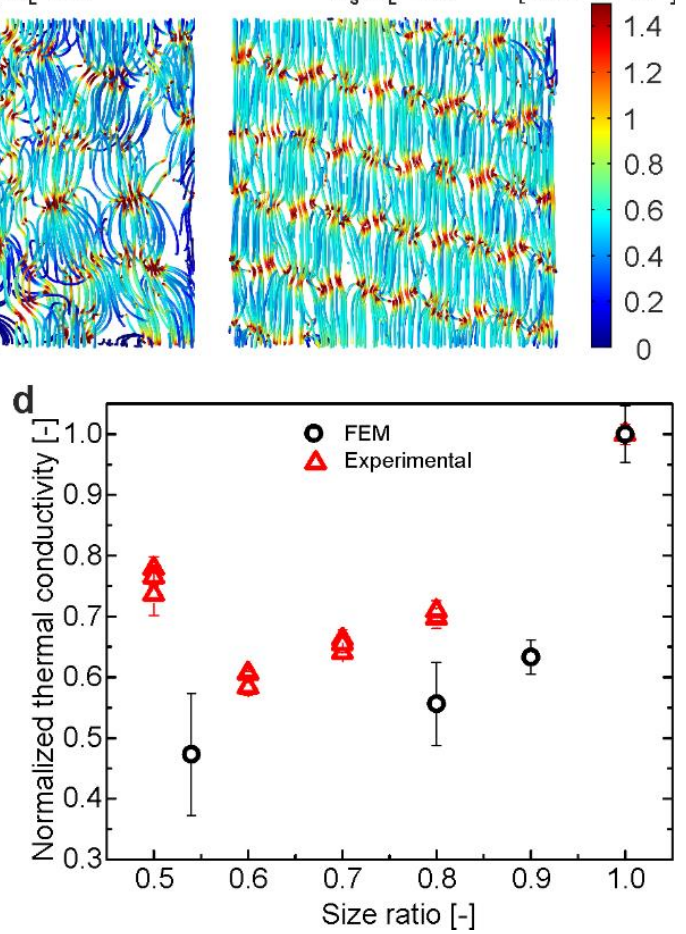

Figure 4. Influence of the size ratio $\left(D_{S} / D_{L}\right)$ on the thermal conductivity at $\sim 20$ vol\%-L. (a) Comparison of the heat flux densities of particles assemblies possessing different size ratios. (b) Histograms of the streamline length and (c) next neighbors per particle of the assemblies. (d) Resulting thermal conductivity from FEM compared to experimental data. 


\section{WILEY-VCH}

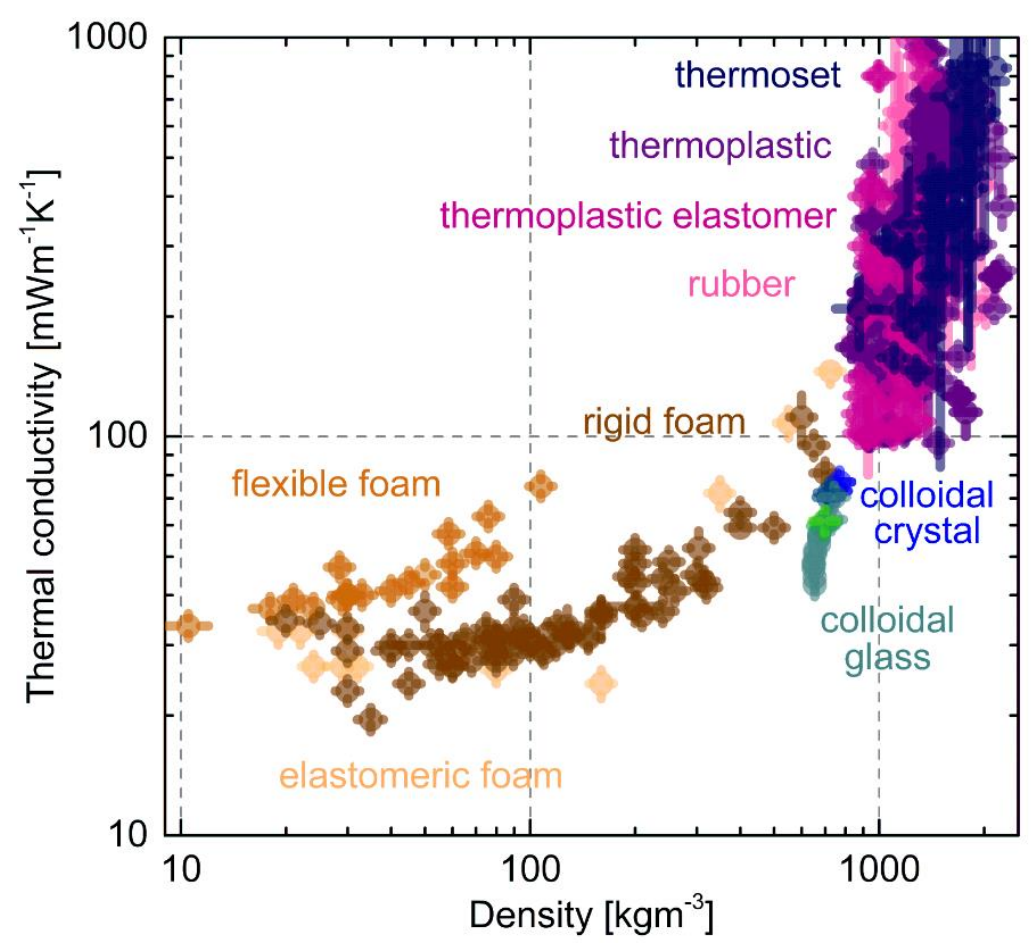

Figure 5. Ashby plot of density vs. thermal conductivity of bulk and foam polymer materials. The introduction of disorder to colloidal ensemble reduces the thermal conductivity (measured in vacuum) while retaining a comparatively high density. 


\section{WILEY-VCH}

The thermal insulation capability of particulate materials can be strongly enhanced by the right degree of disorder. Using binary colloidal assemblies of two differently sized particles as a model platform, a reduction of $\sim 50 \%$ is obtained. This is caused by the elongation of the thermal transport path length, which is governed by the particle structure.

Keyword: colloidal glass; thermal transport; photonic crystal; next neighbor correlation; amorphous limit

Fabian A. Nutz ${ }^{\dagger}$, Alexandra Philipp ${ }^{\dagger}$, Bernd A. F. Kopera, Martin Dulle, Markus Retsch*

Low Thermal Conductivity through Dense Particle Packings with Optimum Disorder

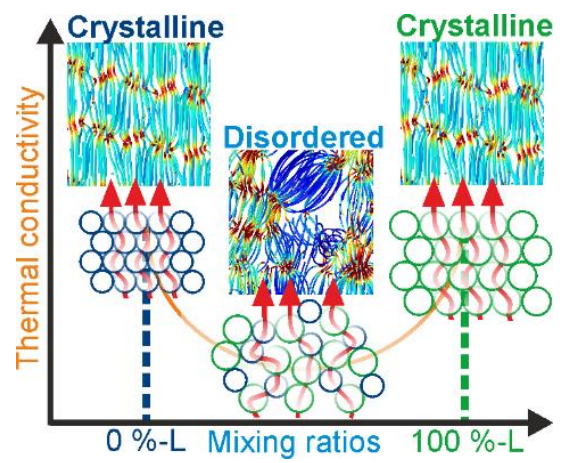




\title{
WILEY-VCH
}

Copyright WILEY-VCH Verlag GmbH \& Co. KGaA, 69469 Weinheim, Germany, 2016.

\section{Supporting Information}

\section{Low Thermal Conductivity through Dense Particle Packings with Optimum Disorder}

\author{
Fabian A. Nutz , Alexandra Philipp ${ }^{\dagger}$, Bernd A. F. Kopera, Martin Dulle, Markus Retsch*
}

\section{Experimental Section}

Materials:

Potassium persulfate (KPS, $\geq 99 \%$, Aldrich), styrene ( $\geq 99 \%$, Aldrich) and acrylic acid (99\%, AA, Aldrich) were used as received. Water was taken from a Millipore Direct Q3UV unit for the entire synthesis and purification steps.

Particle Synthesis: In a typical synthesis, a $500 \mathrm{~mL}$ three-neck flask was charged with $245 \mathrm{~mL}$ milliQ water and $30 \mathrm{~mL}$ of styrene. The mixture was heat to $75^{\circ} \mathrm{C}$ at a stirring speed of $650 \mathrm{rpm}$ and allowed to equilibrate under a slight argon flow for $~ 15 \mathrm{~min}$. Subsequently, $3 \mathrm{~mL}$ of acrylic acid were added, and the mixture was allowed to further equilibrate for $\sim 10 \mathrm{~min}$. To initiate the polymerization, $100 \mathrm{mg}$ KPS, dissolved in $5 \mathrm{~mL}$ milliQ water were rapidly added to the mixture. The polymerization was carried out overnight under a slight argon flow. To adjust the size of the particles the amount of acrylic acid has been varied. ${ }^{[28,}$ ${ }^{29]}$ The reaction was stop by exposing the mixture to ambient atmosphere. The particles were purified by dialysis against milliQ water for five days, changing water twice a day. Binary colloidal self-assembly: Binary colloidal assemblies were fabricated by evaporation-induced self-assembly of a given amount of prior mixed binary particle dispersions in a PTFE beaker. The obtained monoliths generally possess a diameter of $\sim 20 \mathrm{~mm}$ and a thickness of several hundred micrometers.

Dip-coating: Samples for UV-vis measurements were prepared on glass slides by dip-coating $3 \mathrm{wt} \%$ of binary particle solutions. The glass slides were purified with Hellmanex solution an 


\section{WILEY-VCH}

ethanol prior to use. Furthermore, the dip-coating process the glass slides were treated with oxygen plasma to ensure a constant wetting of the particle solutions.

Methods:

Dynamic light scattering: Dynamic light scattering measurements were performed on aqueous particle dispersion at a Malvern Zetasizer with $175^{\circ}$ backscattering geometry to determine the hydrodynamic diameter of the particles and their size distribution

Light microscopy: Bright field light microscopy side view images of the edges of split of the colloidal assemblies were recorded on a Carl Zeiss Axio Imager.A2m light microscope equipped with an AxioCam Icc 1 camera to get a qualitative impression about the long range ordering of the colloidal particles.

Scanning electron microscopy: To determine the hard sphere diameter of the particles, scanning electron microscopy (SEM) was performed on a Zeiss Leo 1530 electron microscope. Therefore, dilute particle dispersions were drop-casted on a silicon wafer and sputtered with $1.3 \mathrm{~nm}$ platinum. Furthermore, SEM side-view images of the edges of split of the colloidal monoliths were obtained to gain an impression of the order within the interior of the monoliths. Fast Fourier transformation was applied on the recorded images using the FFT function, implemented in the software ImageJ 146.r.

Differential scanning calorimetry: Heat capacity determination was carried out according to ASTM E1269 on a TA Instruments Q1000 differential scanning calorimeter under a nitrogen flow of $50 \mathrm{mLmin}^{-1}$ at a heating rate of $20 \mathrm{Kmin}^{-1}$. Two heating cycles were conducted between $-40{ }^{\circ} \mathrm{C}$ and $200{ }^{\circ} \mathrm{C}$. The specific heat capacity was evaluated from the second heating cycle. The mean value from all polystyrene particles under investigation has been used for evaluation (Figure S1). 


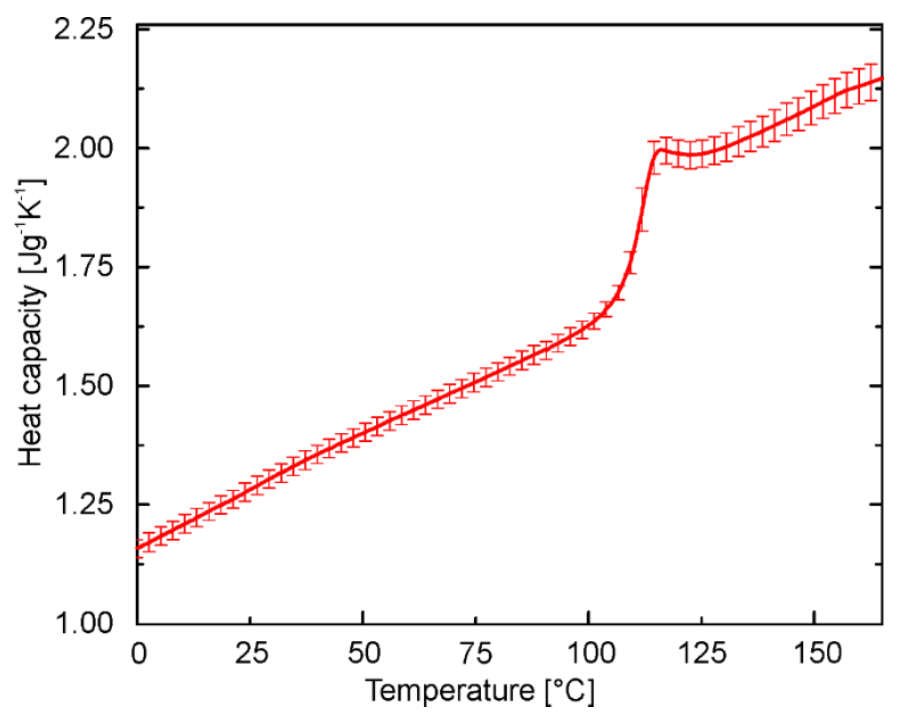

Figure S1. Mean specific heat capacity of the various polystyrene binary assemblies. Error bars represent the standard deviation of the mean value of the specific heat of every mixing ratio.

Density determination: The density of the colloidal assemblies was determined by the volume and mass of the samples. The volume was obtained from a Keyence V3100 3D digital macroscope.

Laser flash analysis: Laser flash analysis was performed on a Linseis XFA 500 XenonFlash apparatus equipped with an InSb infrared detector to obtain the thermal diffusivity of the colloidal assemblies. The thickness of the samples were determined on a Mitotoyo Litematic VL-50. Prior to the measurement, the bottom and top side of the samples were coated with a thin layer of black air-brush color serving as a blocking layer. Furthermore, the samples were coated with a thin graphite layer $(<15 \mu \mathrm{m})$. This enables a good absorbance at the bottom, and high emissivity at the top side of the monoliths. Measurements were performed on at least three individual samples. The standard deviation derives from the average of these individual measurements. Experiments were conducted in vacuum atmosphere at a pressure of $\sim 5 \cdot 10^{-2}$ mbar to exclude any influence of the ambient atmosphere. The raw data was fitted with the radiation fit model provided by the software Aprosoft Laser Flash Evaluation v.1.06. Measurements were performed on at least three samples. 


\section{WILEY-VCH}

A xenon lamp emits a light pulse onto the sample. The thermal energy of the light pulse is absorbed at the bottom graphite layer and travels through the specimen. The thermal energy is then emitted from the top side. This temperature rise is recorded in dependence of the elapsed time since the light pulse by a fast mid-infrared detector. A numerical fitting procedure provided by the commercial software package Aprosoft Laser Flash Evaluation v1.06 based on the one-dimensional temperature diffusion equation was used to determine the thermal diffusivity $\alpha .{ }^{[37]}$ For this, accurate knowledge of the sample thickness is a prerequisite, as the thickness strongly influences half-rise time as given by:

$$
\alpha(T)=\frac{1.38 \cdot d^{2}}{\pi^{2} t_{\frac{1}{2}}}
$$

$t_{1 / 2}$ represents the time needed for the half maximum temperature rise at the top surface. ${ }^{[38]}$ The thermal conductivity $\kappa$ of the sample is calculated by

$$
\kappa(T)=\alpha(T) \cdot c_{p}(T) \cdot \rho(T)
$$

with the specific heat capacity $c_{p}$ and the density $\rho$.

Molecular dynamics (MD) simulations: All dense colloidal assemblies used in the finite element modeling were obtained using LAMMPS. ${ }^{[39]}$ Visualization was done with Ovito ${ }^{[40]}$ Every simulation consisted of 10000 arbitrarily placed spheres of the desired size and number ratio. The simulation box had periodic boundaries and was elongated in the z-direction at the start. This was done to mimic the evaporation of solvent for the real-world system. Using the NPT barostat we compressed the box first along the z-axis up to a pressure where all axes had a comparable length. After that the box was compressed isometrically to a pressure at which 


\section{WILEY-VCH}

no more compression without overlap of the spheres was possible. We used the colloidal Yukawa potential included in the LAMMPS code with a short range repulsive part. Each simulation was run for 60 million steps with a stepsize 0.0001 .

We chose the colloidal Yukawa potential in LAMMPS $^{[41]}$ because it treats the spheres as impenetrable bodies with a fixed radius which prevents unwanted overlap even at very high pressures. The Yukawa potential was necessary in order to facilitate the formation of the $f c c$ phases at the edges of the phase diagram and the real particles also carry a negative net charge. The pairwise interaction is calculated using the formula:

$$
E=\frac{A}{\kappa} e^{-\kappa\left(r-\left(r_{i}+r_{j}\right)\right)} ; \quad r<r_{c}
$$

With $\kappa$ being the screening length (inverse distance units), $r_{i}$ and $r_{j}$ are the radii of the two interacting spheres. The prefactor $A$ has to be specified for each pair type. It is determined from the relationship of the surface charge to surface potential in the presence of an electrolyte. In our case this prefactor is proportional only to the radius of each particle. All other parameter are the same.

We chose a large screening length of 50 which corresponds to $1 / 50$ of the diameter of the large particles in order to hinder crystallization at higher interparticle distances and ensure good mixing of the differently sized particles. As the particles in the experiments are a few hundred nanometers in size this large screening length also approximates the charge interaction distance relative to the particle size. An example of the interaction parameters is given in Table $\mathrm{S} 1$.

Table S1. MD simulation interaction parameters for the size ratio 0.54 .

\begin{tabular}{|c|c|c|c|c|c|}
\hline Pair style & Screening length & Interaction cutoff $\left(\mathbf{r}_{\mathbf{c}}\right)$ & A for 1:1 & A for 1:2 & A for 2:2 \\
\hline Yukawa/colloid & 50 & 1.5 & 10000 & 7500 & 5000 \\
\hline
\end{tabular}




\section{WILEY-VCH}

The analysis of the resulting dense colloidal assemblies was done with a self-written software using $\mathrm{C}++$. The software calculates the radial distribution function $(G(r))$ for the given set of coordinates and uses the first three peaks to determine the three possible next neighbor distances as well as the next neighbor distance cut off. This cut-off was set to $10 \%$ of the particle diameter. In the MD-simulation the type of particle (small or big) is set and the software choses according to this type the appropriate condition to test whether a particle constitutes a next neighbor or not. This enabled us to obtain very detailed information on the local structure around each particle. This procedure also counts particles as next neighbors that are not touching, which is why introduced the additional term "contact neighbors" to distinguish these two sets of neighbors. It also calculates the density as well as the average number of neighbors.

We used a self-written Python script to evaluate the contact area between particles. The script evaluates the number of contacting particles based on the coordinates of the individual spheres. We defined the contact area as the circle created by the intersection of two spherical particles. The area was normalized to the number averaged particle surface of all particles. We increased the size of the original spheres from the MD simulation by a factor of 1.04 in order to make the spheres touch (interpenetrating spheres cannot be simulated by MD). The same scaling factor was used for finite element modeling, and was selected such to give comparable interparticle contact areas to our experimental findings. From this we deduced the contact neighbor histogram. The individual contact areas were further correlated to number of contact neighbors.

Finite element modeling: We used the finite element method (FEM) software COMSOL Multiphysics ${ }^{\circledR}$ to simulate the heat transport through colloidal assemblies. For this, the crystalline (0 and $100 \%$ large particles) and amorphous particle structures (size ratios: 0.54, 0.8 , and 0.9 ) obtained from the MD simulations were used. The particle sizes were adjusted 


\section{WILEY-VCH}

such that the contact areas between adjacent spheres are comparable to the experimental values. All particles were fused into one object and, thus no thermal resistance between the particles was considered. Due to the limited physical memory (128 GB), the system size has been reduced to a feasible size. Therefore, three cubes with around 100 particles were cut out of each invested colloidal assemblies. The mesh size was as chosen to ensure mesh size independent results (see Figure 9c). A temperature difference was imposed to two opposite surfaces of the cubes. Since all remaining surfaces were kept thermally insulating, purely onedimensional thermal transport is obtained. The simulation was conducted along all three directions of the cube (x, y, and z). Using Fourier's law (see Equation S4), the effective thermal conductivity of the assembly is calculated from the computed heat flux, normal to the isothermal faces. Figure demonstrates that three cubes are sufficient to calculate a meaningful average value. Furthermore, heat flux streamlines have been plotted to visualize the heat path length through the different colloidal assemblies. The length of the streamlines $\left(L_{S L}\right)$ has been normalized by the total length of the cube $\left(L_{C}\right)$ to calculate the relative streamline length $\left(L_{S L} / L_{C}\right.$ in $\left.\%\right)$. Figure $\mathrm{S} 10$ demonstrates that three cubes are sufficient to calculate a meaningful average value. Furthermore, heat flux streamlines have been plotted to visualize the heat path length through the different colloidal assemblies. The length of the streamlines $\left(L_{S L}\right)$ has been normalized by the total length of the cube $\left(L_{C}\right)$ to calculate the relative streamline length $\left(L_{S L} / L_{C}\right.$ in $\left.\%\right)$. 


\section{WILEY-VCH}

\section{Comparison between MD simulation and experimental colloidal glass fabrication}

a

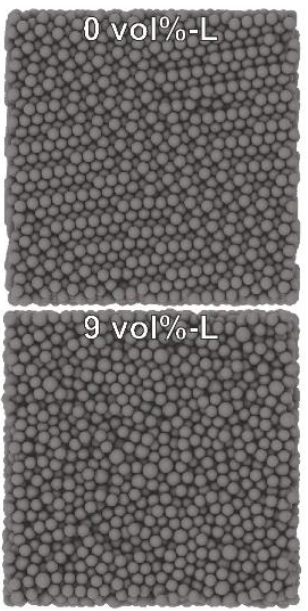

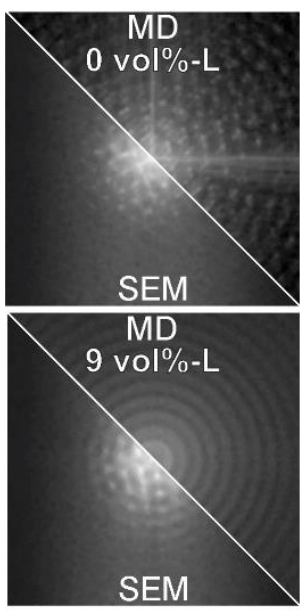

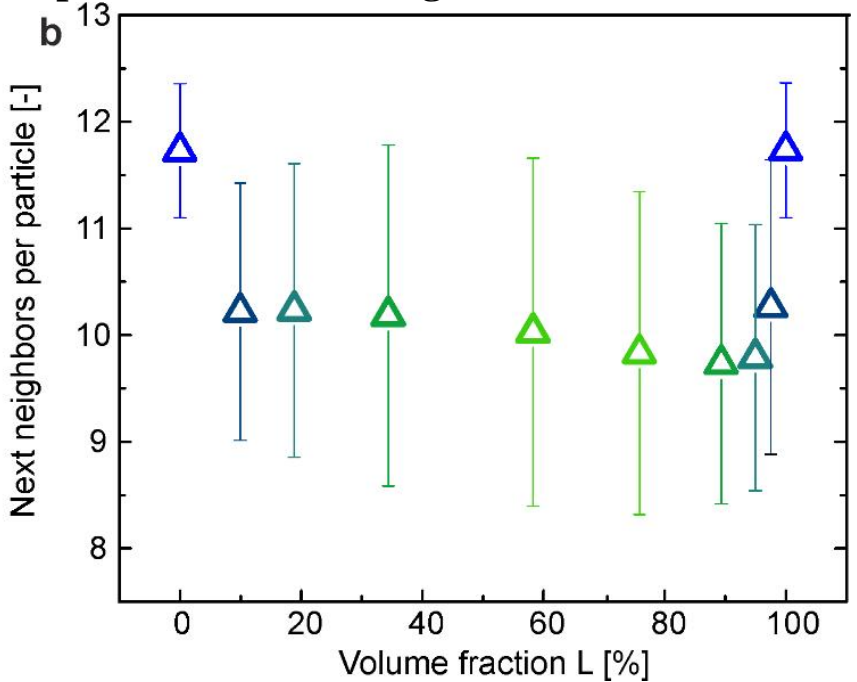

Figure S2. Side views received from MD simulations and number of next neighbors per particles. (a, left) typically obtained particle assemblies from MD studies; (a, right) FFT comparison between experiment and simulation. (b) Average next neighbors per particle $v s$. volume fraction of large particles. Color code indicates ordered (blueish) and disordered (greenish) assemblies.
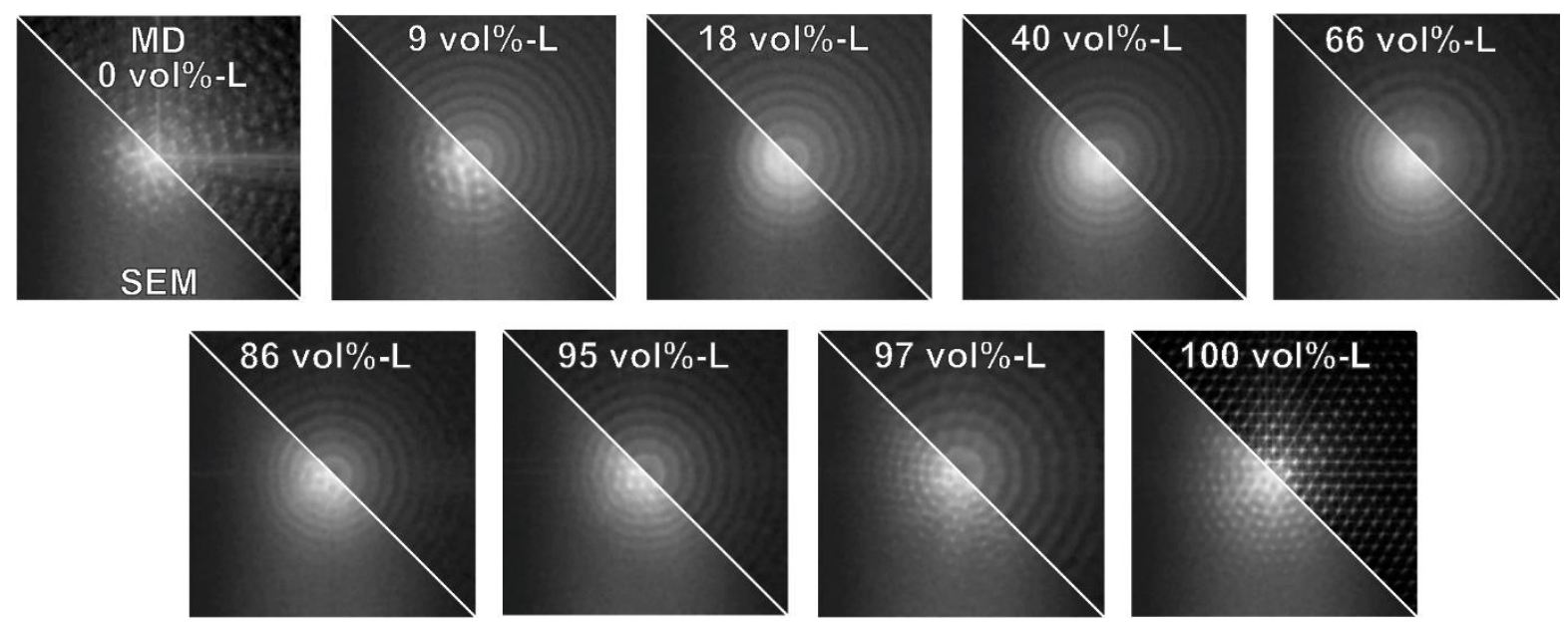

Figure S3. FFT comparison between the particle structures received from MD simulations and SEM side view images. Particularly at low and high mixing ratios a partial crystallinity can be inferred from the experimental SEM images. It indicates an easier colloidal glass formation in the MD simulation compared to the experimental conditions. 


\section{WILEY-VCH}

\section{Influence of mixing ratio on next neighbor distribution function of a size ratio 0.54}
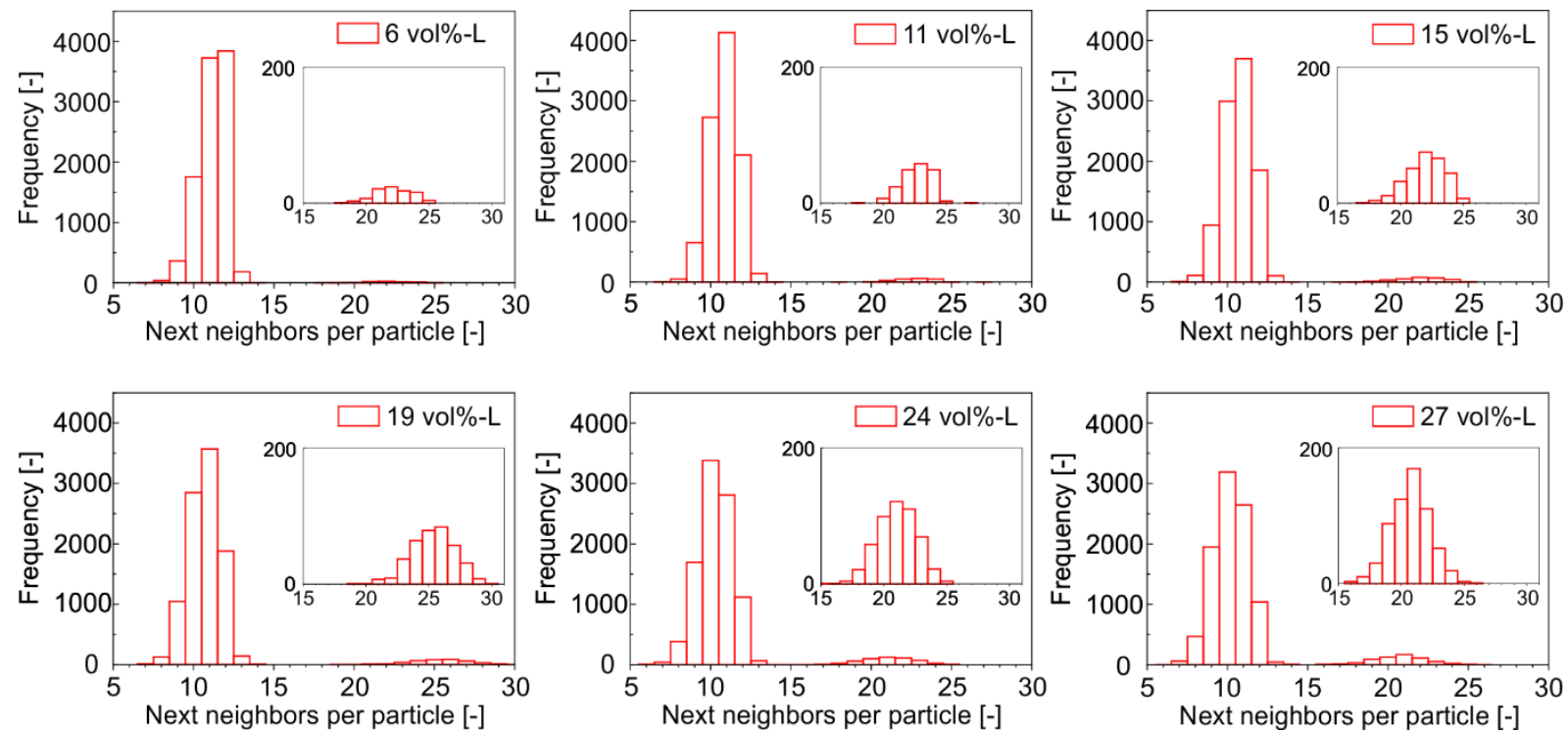

Figure S4. Histograms of the next neighbors per particle for a size ratio of 0.54 . Various particle volume ratios close to the minimum thermal conductivity $(19 \mathrm{vol} \%$-L) are compared. All assemblies have the same average number of next neighbors $(\sim 10-11)$, but only the assembly with $19 \mathrm{vol} \%$-L shows a second fraction with the highest number of next neighbors (20-30). Thus, this assembly is expected to have the lowest thermal conductivity.

\section{Contact neighbor analysis}

a)

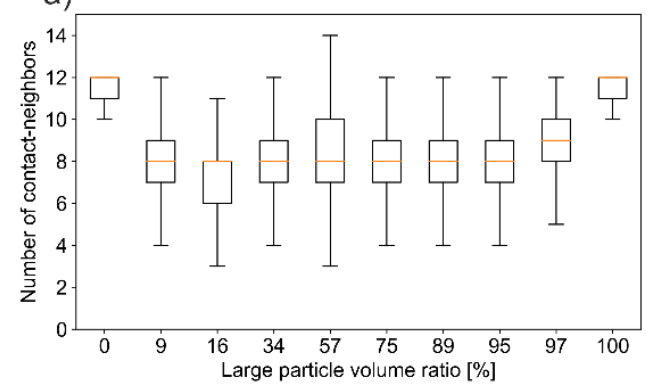

b)

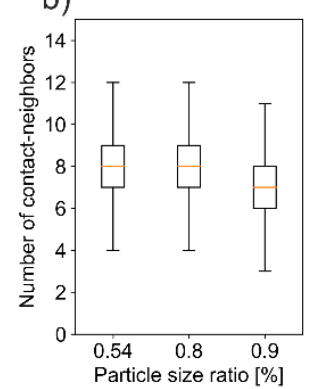

c)

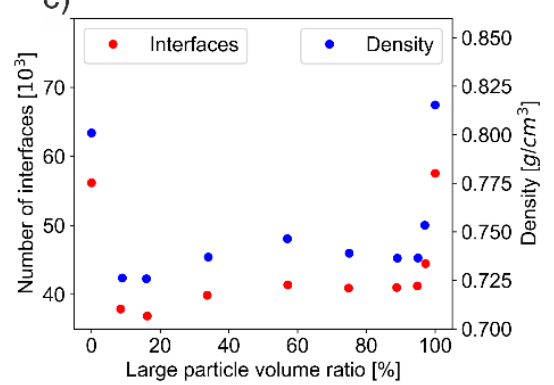

Figure S5. a) Evaluation of the number of contacting next neighbors. An almost constant average number at $\sim 8$ is found with a slight tendency towards fewer contact neighbors for $16 \mathrm{vol} \%$-L. b) Contact neighbor analysis for particle mixture of $\sim 20 \mathrm{vol} \%$-L with different size ratios. c) Total number of interparticle contact areas and effective density in the entire simulation volume. 


\section{WILEY-VCH}

\section{Influence of the interparticle contact area on the thermal conductivity}

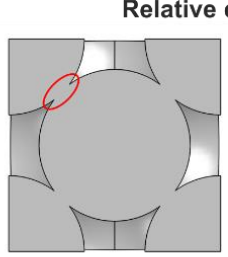

$0.5 \%$
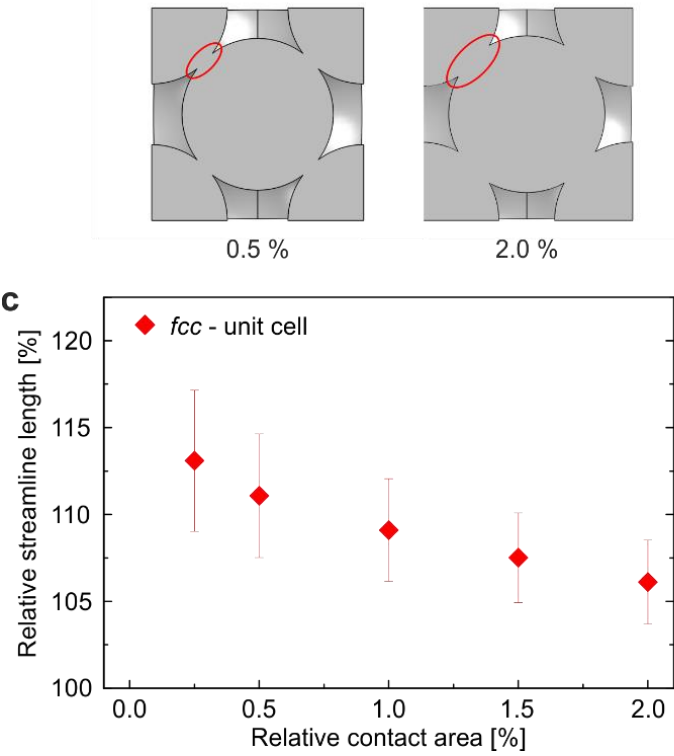

$2.0 \%$ b

contact area:

d

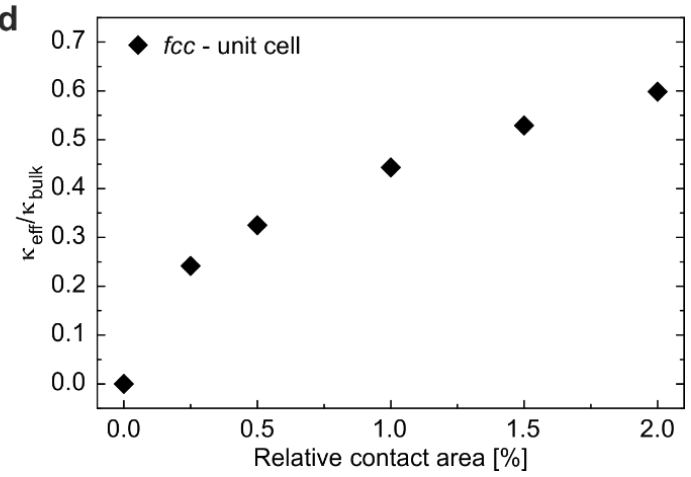

Figure S6. a) Ideal face-centered cubic colloidal crystal with increasing interparticle contact area. b) Heat flux streamlines corresponding to a small $(0.5 \%)$ and large $(2.0 \%)$ contact area. The streamlines bend more strongly in case of $0.5 \%$ contact area. c) Correlation between the interparticle contact area and the streamline length. The relative contact area is the ratio between the circular connection between adjacent particles and the surface area of the complete particle. The total contact area takes the number of next neighbors into account (here 12 for the $f c c$ structure). d) Correlation between the reduced thermal conductivity (relative to the corresponding bulk material) and the relative contact area.

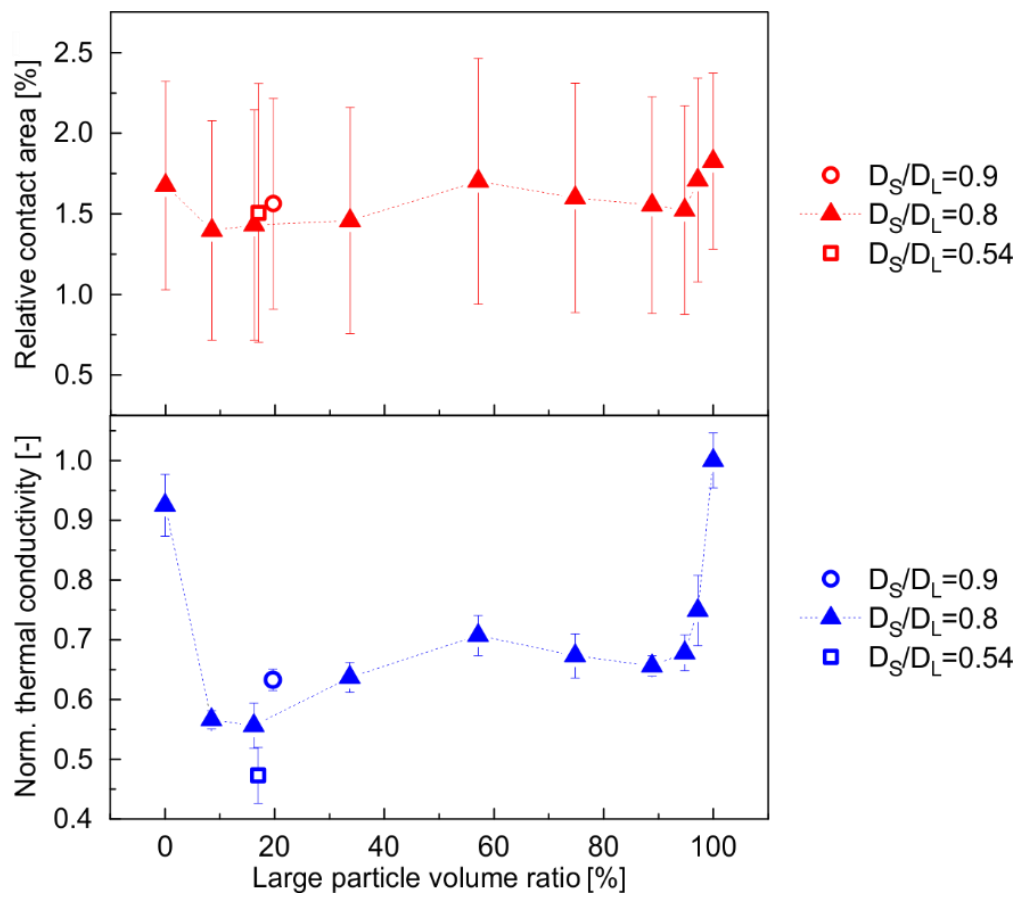

Figure S7. Dependency of normalized thermal conductivity (bottom) and relative contact area (top) on the particle mixing ratio. 


\section{WILEY-VCH}
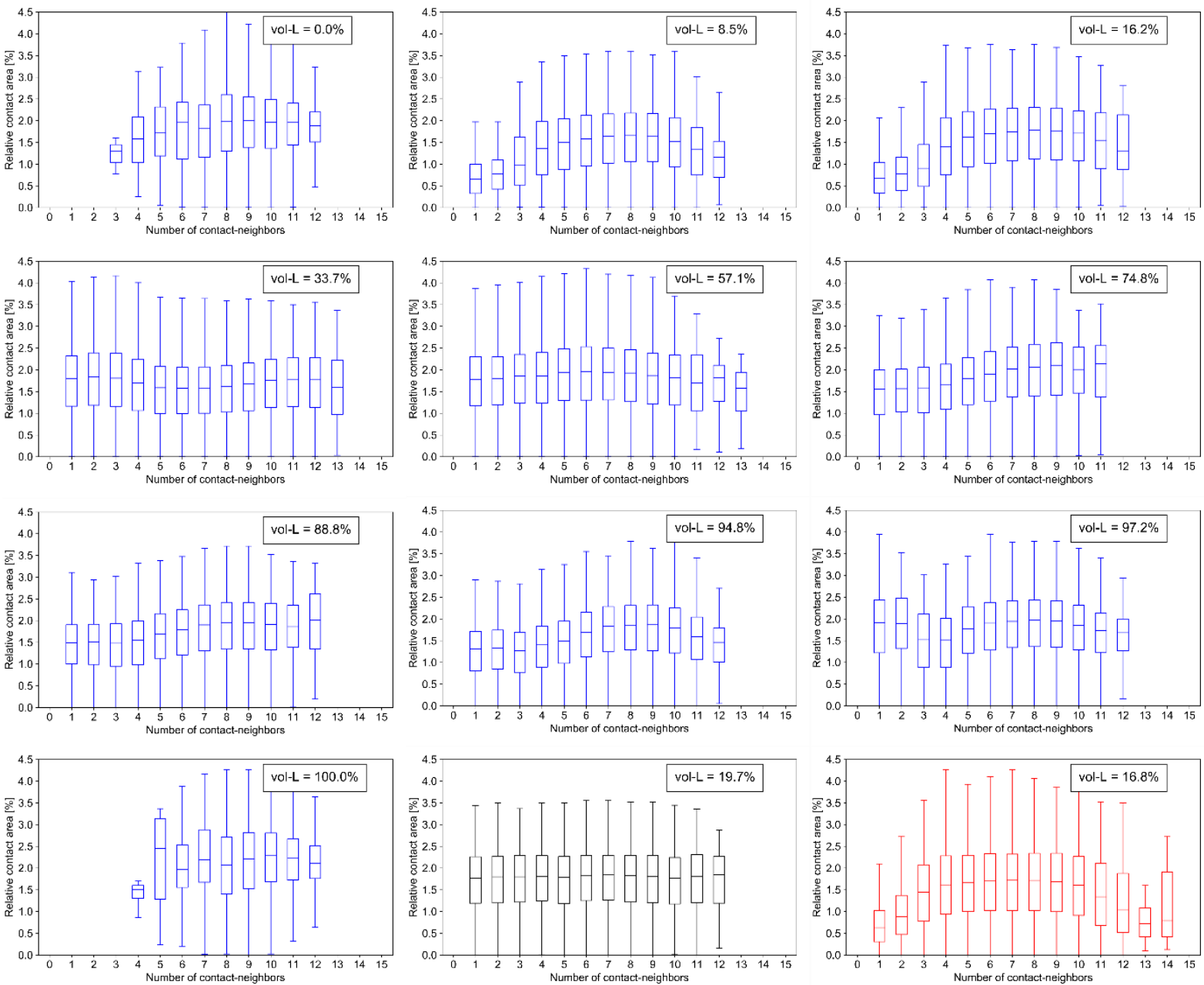

Figure S8. Statistic evaluation of the influence of contacting neighbors on the relative contact area. The box plots give an impression on the average contact are and the width and symmetry of relative contact area distributions. The individual bars at each number of contact neighbors does not include any information on the number of occurrences. The data of size ratio 0.8, 0.9, and 0.54 are colored in blue, black, and red, respectively.

Figure S6 demonstrates the influence of the particle contact area on the streamline length and the effective thermal conductivity for an ideal $f c c$ crystal. Figure S7 shows the average contact area in dependence of the mixing ratio and relates it to our experimental findings. Figure S6 shows that contact area and streamline length indeed depend on one another. A strong reduction, however, is only found for relative small contact areas $<0.5 \%$, which are rarely found in our experiment and simulation. The interparticle constriction adds an additional possibility to increase the thermal path length through the particulate structure. Yet, based on the shown results, we conclude that the changes of the particle contact area play a secondary role, compared to the increase in thermal path length caused by the disordered structure:

a) The relative contact area in our FEM simulation boxes changes in a range from $1.44 \%$ to $1.83 \%$, which can account for a maximum of about $10 \%$ thermal conductivity reduction, when comparing to an $f c c$ reference system. This is much less compared to our findings, when a disordered structure evolves.

b) When looking at the two shallow minima at about 16 vol\%-L and 89 vol\%-L mixing ratio, these turn out to possess rather comparable average contact areas of about $1.5 \%$. The analysis of the contact area distribution (box plots in Figure S8) shows a bell-shaped distribution for the $16 \mathrm{vol} \%$-L case and a decrease towards fewer contact particles for the $89 \mathrm{vol} \%$-L case. 


\section{WILEY-VCH}

The majority of the other mixing ratios exhibit a flat distribution of relative contact areas. (One has to bear in mind that this representation does not give any information on how many particles are present at the respective number of contact neighbors). We assign the more pronounced drop in relative contact area of the $16 \mathrm{vol} \%-\mathrm{L}$ sample to the presence of large particles jammed in a sphere of smaller particles. Hence, the extension of the thermal path length is not only caused by the disordered structure, but additionally aided by an increased constriction around these defect sites.

c) The effect of small particle jamming around larger central spheres becomes even more obvious, when looking at the influence of the size ratio at comparable volume ratios (16$20 \mathrm{vol} \%$-L). The bell-shape of the histogram becomes more pronounced for a decreasing size ratio and shows particularly small relative contact areas for very small and high numbers of contacting neighbors. More similar particle sizes recover a very flat histogram distribution. This is associated with the lack and presence of a high amount of next neighbor spheres, respectively. Consequently, the extension of the thermal path length caused by the introduction of additional next neighbor particles is supported by a stronger constriction around these structures. 


\section{WILEY-VCH}

Finite element modeling

Fourier's law is used to calculate the effective thermal conductivity $\kappa_{e f f}$ :

$$
\kappa_{e f f}=\frac{Q / A}{\Delta T / h}
$$

$Q$ is the heat flow rate normal to the cross-sectional area $A$ of the cube, $\Delta T$ the temperature difference, and $h$ the cube length.

Table S2. Specific heat capacity, density, and thermal conductivity of polystyrene used in FEM simulations.

\begin{tabular}{|c|c|c|}
\hline $\begin{array}{c}\text { Specific heat capacity } \\
{\left[\mathbf{J g}^{-1} \mathbf{K}^{-\mathbf{1}}\right]}\end{array}$ & $\begin{array}{c}\text { Density } \\
{\left[\mathbf{g c m}^{-3}\right]}\end{array}$ & $\begin{array}{c}\text { Thermal conductivity } \\
{\left[\mathbf{m} \mathbf{W m}^{-1} \mathbf{K}^{-1}\right]}\end{array}$ \\
\hline 1.25 & 1.05 & 150 \\
\hline
\end{tabular}
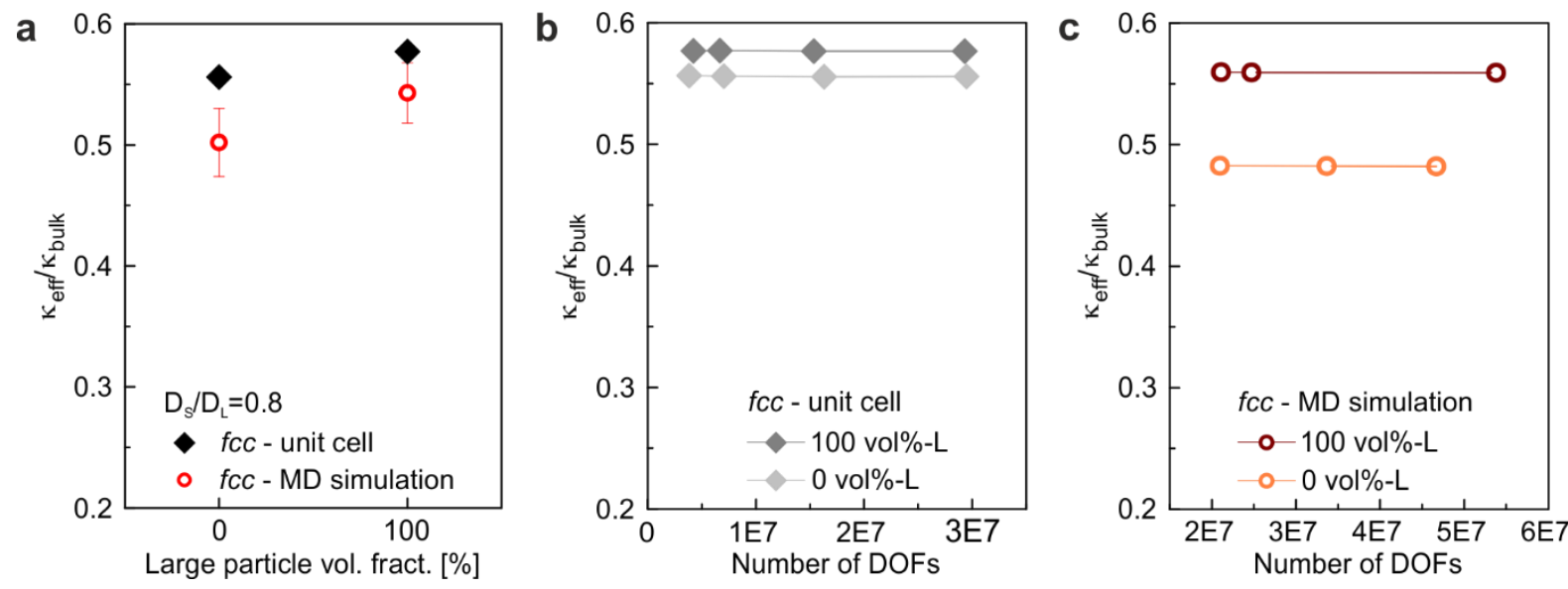

Figure S9. (a) Comparison of the effective thermal conductivity divided by the bulk thermal conductivity of polystyrene obtained for an ideal $f c c$ unit cell and the $f c c$ structure from MD simulation. The average particle contact area of the $f c c$ structure from MD simulation was used to create the unit cell. The slight reduction of the MD simulation compared to the ideal unit cell originates from line and point defects, which are not present in the ideal case. (b, c) The effective thermal conductivity is independent of the number of DOFs and, thus of the mesh size. (c) The data are exemplarily plotted for one position in the $f c c$ structure from MD simulation, respectively. 


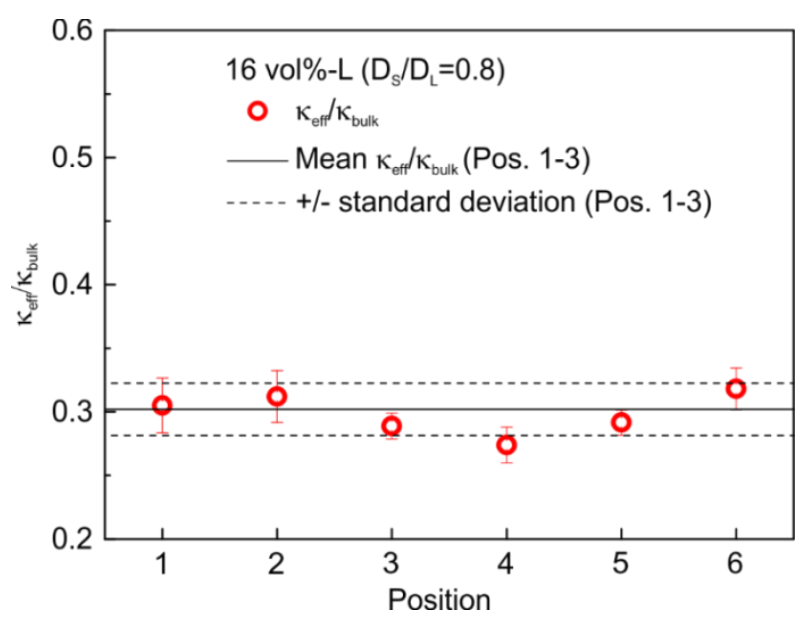

Figure S10. Effective thermal conductivity of the particle assembly with 16 vol\% large particles (size ratio 0.8) obtained for six cubes cut out of different positions of the amorphous particle structure (red circles). The error bars arise from the three different simulation directions per cube (x,y, and $\mathrm{z}$ ). The black solid line represents the mean effective thermal conductivity calculated from the first three values, the dashed line is the corresponding standard deviation. This plot shows that three positions are sufficient to calculate a meaningful average value.

\section{References}

[1] J. W. Goodwin, J. Hearn, C. C. Ho, R. H. Ottewill, Colloid. Polym. Sci. 1974, 252, 464.

[2] R. H. Ottewill, J. N. Shaw, Kolloid Z. Z. Polym. 1967, 215, 161.

[3] S. Plimpton, J. Comput. Phys. 1995, 117, 1.

[4] S. Alexander, Modell. Simul. Mater. Sci. Eng. 2010, 18, 015012.

[5] L. Dusza, High Temp. - High Press. 1995, 27, 467.

[6] W. J. Parker, R. J. Jenkins, C. P. Butler, G. L. Abbott, J. Appl. Phys. 1961, 32, 1679.

[7] S. Samueal A, Statistical Thermodynamics of Surfaces, Interfaces, and Membranes, Westview Press, Virgina, US, 2003, 284. 